\title{
Preparation and Optimization of Graphene Pure Water Film Based on Hummers Improved Method
}

\author{
Ning Xue \\ Resource and Material College, Northeastern University at Qinhuangdao, Qinhuangdao, China \\ Email: 1004809474@qq.com
}

How to cite this paper: Xue, N. (2020) Preparation and Optimization of Graphene Pure Water Film Based on Hummers Improved Method. Open Access Library Journal, 7: e6493.

https://doi.org/10.4236/oalib.1106493

Received: June 2, 2020

Accepted: July 11, 2020

Published: July 14, 2020

Copyright $\odot 2020$ by author(s) and Open Access Library Inc.

This work is licensed under the Creative Commons Attribution International License (CC BY 4.0).

http://creativecommons.org/licenses/by/4.0/

\section{(c) Open Access}

\begin{abstract}
In this experiment, graphene oxide was prepared by Hummers' improved method, and water-soluble graphene oxide was prepared into graphene pure water film with a thickness of only 1 micron through pressure filtration. The thickness and densification of graphene membranes were controlled by improving pressure conditions and adjusting reduction degree, so as to achieve the expected nanofiltration grade of graphene pure water membranes that can be used to purify water. The graphene pure water film was successfully prepared, water pressure infiltration and pollution resistance experiments were carried out, and the preparation process was optimized by combining the experimental data.
\end{abstract}

\section{Subject Areas}

Functional Materials

\section{Keywords}

Graphene Oxide, Pure Water Membrane, Hummers Improvement Method, Penetration

\section{1. 概述}

石墨烯近年来在材料科学研究领域成为大热点, 氧化石墨烯 (Graphene Oxide, GO)在膜材料领域备受关注。虽然目前氧化石墨烯的结构难以详细、 准确地描述, 但研究者们普遍接受的结构模型是 GO 单片上随机分布着羟基 和环氧基, 而在单片的边缘则引入了羧基和羰基 [1]。这些分布的亲水性的官 能团为研究者们提供了利用 GO 进行膜表面改性的思路。李瑞云等人利用氧 
化石墨烯和经还原的氧化石墨烯共混入 PVDF 铸膜液中, 然后用相转化的方 法进行超滤膜的制备, 研究结果显示该法制得的 PVDF 膜比原膜在亲水性、 透水性等方面表现更加出色; Lee 等人在采用相同的手段, 利用 PS 制备超滤 膜的试验中得到了相似的结果, 并在 MBR 小试装置的运行中也表明 GO 功能 化 PS 超滤膜的抗污染性能更优越[2]。Isloor 等人在研究中发现, 在聚砜铸膜 液中添加 $\mathrm{GO}$ 可以提高膜的亲水性能, $\mathrm{Na}_{2} \mathrm{SO}_{4}$ 的截留率可提高至 $74 \%$ [3]; 而 $\mathrm{Xu}$ 等人则把有机硅功能化的 GO 与 PVDF 进行共混制膜, 制成的超滤膜在机 械强度上、通量上均有优异表现, 由于内差极化是影响正渗透过程的主要因 素之一, GO 优化了多孔的支撑层底膜, 从而减小了浓差极化现象的影响, 对于膜性能的提高具有重要的意义。GO 为片状结构, 嵌于聚合物中使得聚 合物表面呈现盾鳞状结构 [4], 而且长度较短且形态坚固, 从结构和化学理论 上来说, GO 是完全可以优化复合膜的表面或底膜支撑层的亲水性和微观形 貌, 使膜表面或膜孔道内壁处于亲水性状态, 能够有效地减缓污染物的吸附 和减少内部浓差极化的影响[5]。

石墨烯纯水膜是在已有的石墨烯掺杂改性研发膜基础上提出的一种新型 的膜材料结构。通过将氧化石墨烯或还原性的氧化石墨烯通过压滤、抽滤等 方式, 将石墨烯进行有序的堆叠, 形成具有特定间隙厚度的石墨烯纯水膜[6]。 而石墨烯本身的机械结构强度以及可高达 $2630 \mathrm{~m}^{2} / \mathrm{g}$ 的比表面积, 为石墨烯水 渗透能力提供了一定保障[7]。

\section{2. 实验设备与实验方法}

\section{1. 实验设备}

本实验采用的是型号 HP4750 的耐高压渗透池作为主要压滤设备, 如图 1 所示。

\section{2. 实验方法}

1) 氧化石墨烯制备: 本实验制备的 GO 采用化学氧化法。化学氧化法最 初由 Brodie 于 1860 年提出, 在发烟硝酸的环境中, 以 $\mathrm{KClO}_{3}$ 为氧化剂, 对 本体石墨进行氧化。此后, Staudenmaier 提出另一种氧化方法, Akasay 小组 对该方法进行了改进, 即在浓硫酸和浓硝酸的混合酸中, 以 $\mathrm{KClO}_{3}$ 为氧化剂, 对本体石墨进行氧化处理, 最后以稀盐酸和水洗涤。目前最为常用的是 Hummers 法, 此方法通过将石墨粉和无水硝酸至于冰浴内的浓硫酸中, 以高
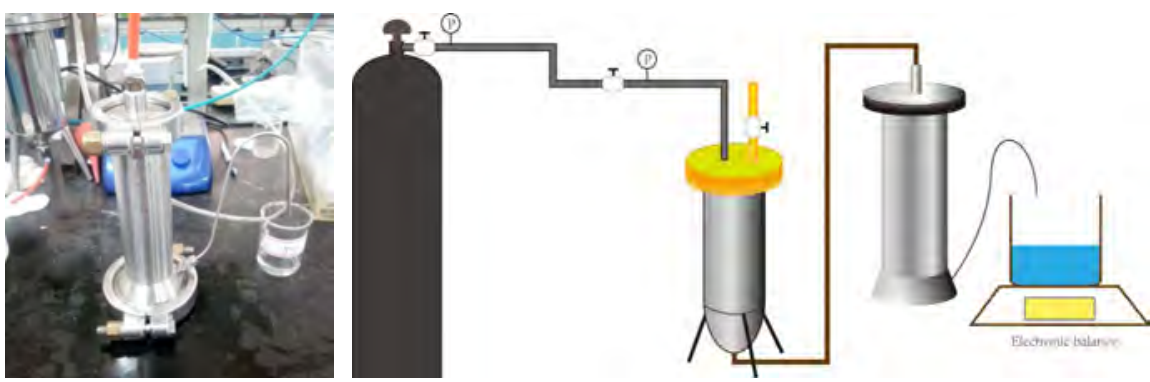

图 1. HP4750 耐高压渗透池 
锰酸钾为氧化剂进行氧化处理, 用浓度为 $30 \%$ (质量分数)的双氧水还原剩余 的氧化剂, 最后洗涤, 过滤, 真空脱水得到氧化石墨烯, 并且 Hummers 法表 明, 得到的氧化石墨烯含氧量比较高时呈现黄色, 含氧量低时则呈现黑色。

为制备具有特殊性能的石墨烯产物, 本实验综合考虑各种研究, 我们所 采用的方法是在第一阶段中, 我们对天然石墨粉进行预氧化, 然后 Hummers 法处理。在这项研究中使用的方法如下:

a) 石墨粉、过硫酸钾, 五氧化二磷加入到 $10-50 \mathrm{ml}$ 浓硫酸中在 $80^{\circ} \mathrm{C}$ 水 浴搅拌反应 $6 \mathrm{~h}$, 用 $2000 \mathrm{ml}$ 去离子水稀释, 然后用 $0.22 \mu \mathrm{m}$ 滤膜过滤, 自然 干燥。

b) 将预氧化产物加入到 $0^{\circ} \mathrm{C} 300 \mathrm{ml} 98 \%$ 浓硫酸, 搅拌下缓慢加入 $3 \mathrm{~g}$ 硝 酸钠和 $35 \mathrm{~g}$ 高锰酸钾, 保持温度低于 $20^{\circ} \mathrm{C}$, 紧接着, 反应液在 $35^{\circ} \mathrm{C}$ 下应 $4 \mathrm{~h}$, 用 $1 \mathrm{~L}$ 去离子水稀释, 保持温度低于 $50^{\circ} \mathrm{C}$, 再加 $1000 \mathrm{ml}$ 去离子水, 并 逐滴加入过氧化氢, 等溶液变澄清后, 趁热过滤反应液, 用 $1: 10$ 的盐酸洗涤, 再用去离子水充分洗涤, 室温干燥, 透析一周。最后于冷冻干燥机中冻干。

2) 实验仪器: 超声仪、烘箱、烧杯玻璃棒若干。

3) 实验材料: 氧化石墨烯(本次实验直接使用已经制备成功的氧化石墨 烯粉末)。

4) 具体步骤:

a) 将氧化石墨烯分散溶解在去离子水中。

b）超声仪超声分散有氧化石墨烯的水溶液。

c) 将分散开的氧化石墨烯置于高压渗透池, 进行压制。

\section{3. 实验制备样品图}

本实验制备样品如图 2 和图 3 所示。

\section{4. 膜的测试}

\section{氧化石墨烯的 SEM 图}

图 4 所示是压制的石墨烯纯水膜的 SEM 图。膜表面没有均匀的孔隙, 面 内有褶皱状凸起, 凸起的部分不明显。面内还存在部分线状和颗粒状, 这可 能是膜上石墨烯边缘褶皱部分引起的。图右是膜的断面图, 初步测量膜的厚 度在 $100-200$ 纳米之间。

\section{5. 总结}

本文通过 Hummers 改进法制备氧化石墨烯, 将水溶性的氧化石墨烯通过 压滤的方式, 制备成厚度仅为 1 微米的石墨烯纯水膜。实验通过改进压力条 件和调节还原程度控制石墨烯膜的厚度和致密程度, 达到预期的纳滤级别的 可用于净化水质的石墨烯纯水膜, 并通过 SEM 对氧化石墨烯及膜进行简单的 表征。具体如下:

1) 本实验初期进行了氧化石墨烯的制备, 在此基础上完成了石墨烯纯水 膜的制备; 


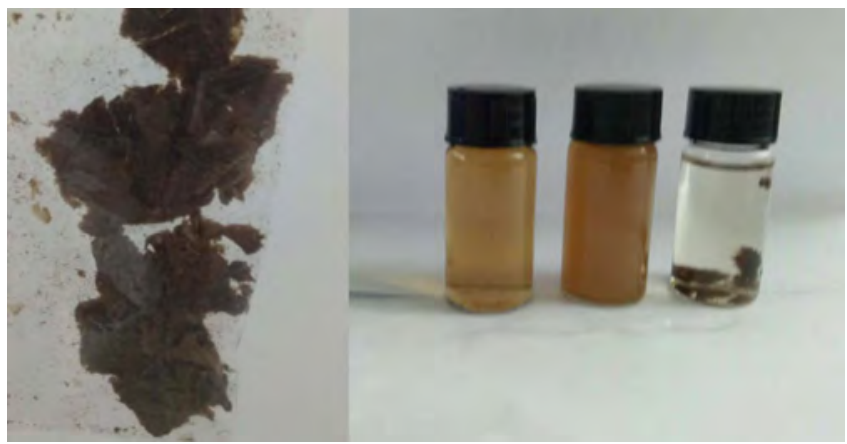

图 2. 左为压制的氧化石墨烯纯水膜。右为氧化石墨烯、石墨 烯溶解于去离子水中的示意图, 溶液呈黄色澄清溶液

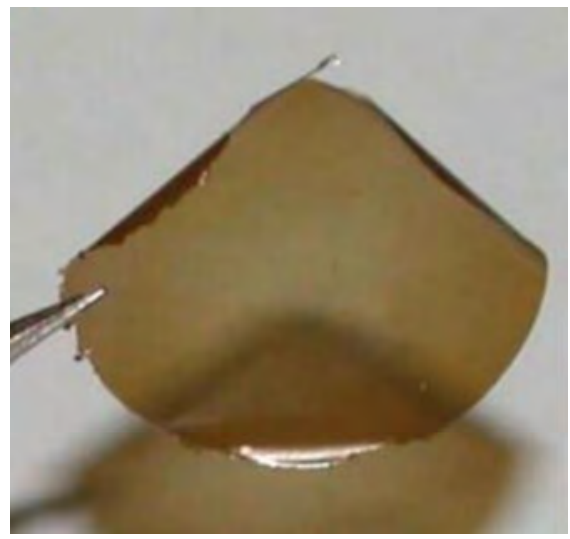

图 3. 最终固化成型氧化石墨烯纯水膜
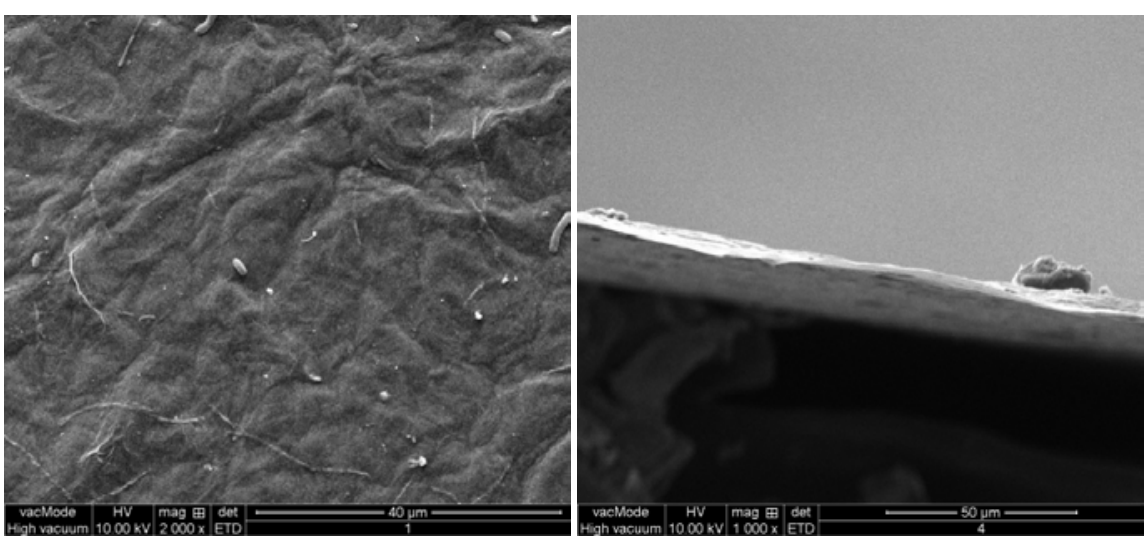

图 4. 石墨烯纯水膜的 SEM 图

2) 膜的基本测试通过 SEM 对氧化石墨烯及膜进行简单的表征;

3) 膜的渗透测试由于时间问题，将在后期进行完善;

4) 本次实验的制备方法还有进一步改性的空间, 在设备的使用和材料的 选择上还有改进的空间;

5) 在未来的实验开展中, 将进一步加入金属元素，进行石墨烯纯水膜的 性能测试, 对膜的性能进行改良与优化, 以应用到更加广泛的领域中, 为后 续的石墨烯应用提供了一定的思路与借鉴。 


\section{Conflicts of Interest}

The author declares no conflicts of interest regarding the publication of this paper.

\section{References}

[1] 曹晓春. 聚偏氟乙烯与纳米二氧化钛复合超滤膜的研制[D]: [博士学位论文]. 哈 尔滨: 哈尔滨工业大学, 2006.

[2] 问国航, 胡祖明, 于俊荣, 王彦, 诸静. PMIA/PDAPs 复合超滤膜的制备与性能研 究[J]. 合成纤维工业, 2019, 42(4): 24-29.

[3] 肖建平. 中空纤维超滤膜的有机污染及清洗方法研究 [D]: [硕士学位论文]. 重庆: 重庆大学, 2004.

[4] 洪金成, 李卫星, 邢卫红. GO/PVDF 超滤膜的表征及处理造纸废水的过滤特性 [J]. 南京工业大学学报: 自然科学版, 2015, 37(2): 39-44.

[5]王闪闪, 王全杰, 曲家乐. 不同还原程度氧化石墨烯-水性聚氨酯共混膜的性能 研究[J]. 炭素技术, 2013, 32(4): 23-26.

[6] 李瑞云. 氧化石墨烯对聚合物分离膜的亲水改性研究[D]: [硕士学位论文]. 大连: 大连理工大学, 2012.

[7] Hegab, H.M. and Zou, L. (2015) Graphene Oxide-Assisted Membranes: Fabrication and Potential Applications in Desalination and Water Purification. Journal of Membrane Science, 484, 95-106. https://doi.org/10.1016/j.memsci.2015.03.011 